\title{
Performance analysis and power control for full-duplex relaying with large-scale antenna array at the destination
}

\author{
Liang $\operatorname{Han}^{1,2^{*}}$, Bin Shi ${ }^{2}$ and Weixia Zou ${ }^{3}$
}

\begin{abstract}
Full-duplex relaying can increase the spectral efficiency by allowing the relay to transmit and receive at the same time and over the same frequency. To overcome the challenge that the destination treats the source-destination link as interference, we employ large-scale antenna array at the destination. By using the fact that the source-destination channel vector and relay-destination channel vector become pairwise orthogonal, the source-destination link can be converted to useful signal. We first derive the achievable rates and outage probabilities for direct transmission, half-duplex relaying, and full-duplex relaying, respectively. Two relaying protocols, i.e., amplify-and-forward (AF) and decode-and-forward (DF), are considered. Then, based on the channel state information (CSI) availability, the optimal transmit power of the full-duplex relay node is obtained by maximizing the achievable rates or minimizing the outage probabilities of full-duplex relaying. Numerical results show that the full-duplex relaying performs better than half-duplex relaying when the residual self-interference is small, and the performance of full-duplex relaying can be improved by using power control.
\end{abstract}

Keywords: Full-duplex relaying, Amplify-and-forward, Decode-and-forward, Achievable rate, Outage probability, Power control

\section{Introduction}

In recent years, the fourth-generation (4G) mobile communication system has been deployed and used worldwide. However, with an explosion of wireless devices and services, there is growing demand for data traffic that cannot be accommodated by 4G. Therefore, the fifthgeneration (5G) mobile communication system, which is expected to be commercially available by 2020 , has become a hot research topic in both academia and industry $[1,2]$.

As one of the most promising technique for the $5 \mathrm{G}$ mobile communication system, large-scale antenna array or massive multiple-input multiple-output (MIMO) has drawn significant research interests [3-6]. The concept of massive MIMO was first proposed by Marzetta [7],

*Correspondence: hanliang_tjnu@163.com

${ }^{1}$ Tianjin Key Laboratory of Wireless Mobile Communications and Power Transmission, Tianjin Normal University, Tianjin 300387, China

${ }^{2}$ College of Electronic and Communication Engineering, Tianjin Normal University, Tianjin 300387, China

Full list of author information is available at the end of the article showing that by employing unlimited number of antennas at a base station (BS), the effect of thermal noise and small-scale fading can be averaged out, the channel vectors between the users and the BS become pairwise orthogonal, and the inter-user interference can be eliminated. In [8], the authors compared two linear precoding schemes, i.e., conjugate beamforming and zero forcing (ZF), with respect to spectral efficiency and energy efficiency in a single-cell downlink scenario, and showed that for high spectral efficiency and low energy efficiency, ZF outperforms conjugate beamforming, while at low spectral efficiency and high energy efficiency, the opposite holds. In [9], the transmit power scaling laws and lower capacity bounds for maximum ratio combining (MRC), $\mathrm{ZF}$, and minimum mean-square error (MMSE) detection were derived. It was shown that when the number of BS antennas $M$ grows without bound, the transmitted power of each user could be reduced proportionally to $M$ if the BS has perfect channel state information (CSI) and $1 / M$ if CSI is estimated from uplink pilots. In [10], the authors 
investigated how massive MIMO performs in real propagation environments and showed that the performance is close to that in independent and identically distributed (iid) Rayleigh channels, so the theoretical advantages of massive MIMO can be obtained in real channels.

On the other hand, encouraged by the advance in selfinterference cancelation (SIC) algorithms [11, 12], fullduplex relaying, which can transmit and receive at the same time and over the same frequency, has also attracted great research interests. In [13], the authors proposed a full-duplex relay system which shares the time resource as well as the antennas at the relay node and eliminates the underlying interferences using precoding. In [14], the exact outage probability of full-duplex decodeand-forward (DF) relay systems was derived by considering the self-interference as a Rayleigh fading channel. It was shown that the full-duplex relaying is superior to half-duplex relaying in terms of the outage probability as the signal-to-interference ratios (SIRs) are higher and the signal-to-noise ratio (SNR) is lower. Riihonen et al. derived an optimal gain control scheme for full-duplex amplify-and-forward (AF) relaying which considered the effect of residual loop interference in [15], investigated three spatial domain SIC solutions for full-duplex MIMO relay systems in [16], and proposed a hybrid relaying which could switch between full-duplex and half-duplex mode in [17]. Reference [18] derived the outage performance of multi-hop full-duplex relaying by considering all the inter-relay interference and echo-interference. Reference [19] investigated the power and location optimization for full-duplex DF relay systems. From the above references, it is clear that there are two main challenges facing the use of full-duplex relaying. The first challenge is how to mitigate or cancel the self-interference at the relay due to signal leakage between the relay transmit and receive antenna [12-19], and the second challenge is how to deal with the signal from the source since the destination always treats the direct link from the source to the destination as interference [14, 17-19].

Since low complexity linear signal processing would achieve near optimal performance in massive MIMO systems, some researchers have introduced massive antennas into full-duplex relay systems. In [20], the authors investigated a multi-pair full-duplex relay system, where the relay is equipped with massive arrays and uses $\mathrm{ZF}$ and MRC/MRT to process the signals, and showed that the large number of spatial dimensions available can be effectively used to suppress the loop interference in the spatial domain. In [21], the authors studied the hardware impairments aware transceiver design for massive MIMO full-duplex relaying, where multiple source-destination pairs communicate simultaneously with the help of a fullduplex relay equipped with very large antenna arrays. Note that these works assume the relay is equipped with massive arrays, and there is no work considering the destination is equipped with massive arrays in full-duplex relay systems.

In this paper, we investigate the performance of fullduplex AF and DF relaying with large-scale antenna array at the destination. Since the source-destination channel vector and relay-destination channel vector become pairwise orthogonal, the destination can treat the sourcedestination link as useful signal and therefore the performance can be improved. Furthermore, we optimize the transmit power of the full-duplex relay node by maximizing the achievable rates or minimizing the outage probabilities.

\section{System model}

We consider a three-node relay channel, where a singleantenna source communicates with an $N$-antenna destination through a full-duplex relay, as depicted in Fig. 1. We assume the relay is equipped with two antennas, one for receiving and the other for transmitting. In cellular networks, the source can be a user and the destination can be a BS.

Denote the channel of the source-relay, sourcedestination, and relay-destination links as $h_{\mathrm{sr}}, \mathbf{h}_{\mathrm{sd}} \in \mathbb{C}^{N \times 1}$, and $\mathbf{h}_{\mathrm{rd}} \in \mathbb{C}^{N \times 1}$, respectively, which are modeled to be frequency flat and quasi static. All the channel elements are assumed to be zero-mean complex Gaussian random variables (Rayleigh fading), and the variance for $h_{\mathrm{sr}}$, each element of $\mathbf{h}_{\text {sd }}$, and each element of $\mathbf{h}_{\mathrm{rd}}$ are given as $\eta_{\mathrm{sr}}, \eta_{\mathrm{sd}}$, and $\eta_{\mathrm{rd}}$, respectively. Since the self-interference cannot be completely canceled, we denote the residual self-interference channel as $h_{\mathrm{rr}}$, which is also assumed to be zero-mean complex Gaussian random variables with variance $\eta_{\mathrm{rr}}$.

The transmit signals from the source and the relay in time slot $t$ are denoted as $x_{\mathrm{S}}(t)$ and $x_{\mathrm{r}}(t)$, respectively. We assume the transmit power of the source and the relay are $P_{\mathrm{s}}$ and $P_{\mathrm{r}}$, respectively, i.e., $E\left[\left|x_{\mathrm{s}}(t)\right|^{2}\right]=P_{\mathrm{s}}$ and $E\left[\left|x_{\mathrm{r}}(t)\right|^{2}\right]=P_{\mathrm{r}}$.

\subsection{Direct transmission}

For the direct transmission, the source transmit the signal directly to the destination. In time slot $t$, the received signal at the destination is given as

$$
\mathbf{y}_{\mathrm{d}}(t)=\mathbf{h}_{\mathrm{sd}} x_{\mathrm{s}}(t)+\mathbf{n}_{\mathrm{d}}(t),
$$

where $\mathbf{n}_{\mathrm{d}}(t)$ denotes the complex additive white Gaussian noise (AWGN) at the destination with zero-mean and covariance matrix $E\left[\mathbf{n}_{\mathrm{d}}(t) \mathbf{n}_{\mathrm{d}}(t)^{H}\right]=\sigma_{\mathrm{d}}^{2} \mathbf{I}_{N}$.

\subsection{Half-duplex relaying}

For the half-duplex relaying, the transmission is carried out in two time slots. In the time slot $t$ (assumed to be 


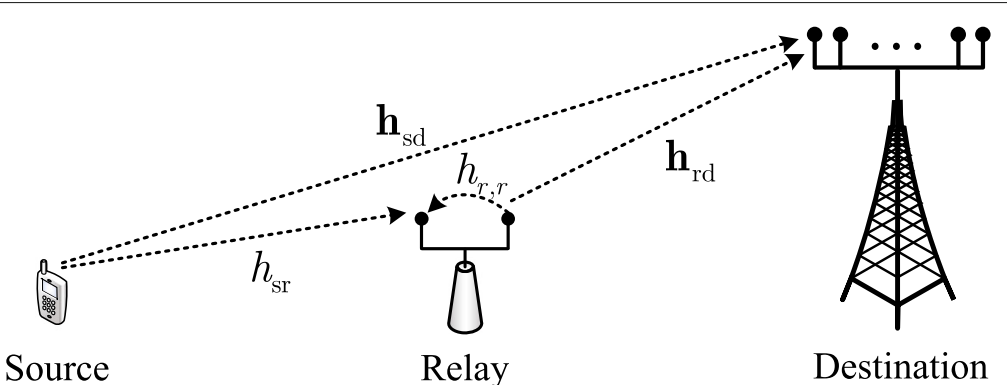

Fig. 1 System model for full-duplex relaying with large-scale antenna array at the destination

odd), the source transmits a signal to the relay and the destination. The received signal at the destination is the same as (1), and the received signal at the relay is given as

$$
y_{\mathrm{r}}(t)=h_{\mathrm{sr}} x_{\mathrm{s}}(t)+n_{\mathrm{r}}(t),
$$

where $n_{\mathrm{r}}(t)$ denotes the AWGN at the relay with zeromean and covariance $\sigma_{\mathrm{r}}^{2}$. In the time slot $t+1$, the relay transmit a signal to the destination, and the received signal at the destination is given as

$$
\mathbf{y}_{\mathrm{d}}(t+1)=\mathbf{h}_{\mathrm{rd}} x_{\mathrm{r}}(t+1)+\mathbf{n}_{\mathrm{d}}(t+1) .
$$

When the AF protocol is used, the relay amplifies the received signal $y_{\mathrm{r}}$ by a scaling factor $\beta$, so the transmit signal at the relay is $x_{\mathrm{r}}(t+1)=\beta y_{\mathrm{r}}(t)$. To satisfy the instantaneous transmit power at the relay, the scaling factor is chosen as

$$
\beta=\sqrt{\frac{P_{\mathrm{r}}}{P_{\mathrm{s}}\left|h_{\mathrm{sr}}\right|^{2}+\sigma_{\mathrm{r}}^{2}}} .
$$

When the DF protocol is used, the relay decodes its received signal and checks if it is correct. If the relay can decode the received signal successfully, the transmit signal at the relay is $x_{\mathrm{r}}(t+1)=\sqrt{\frac{P_{\mathrm{r}}}{P_{\mathrm{s}}}} x_{\mathrm{s}}(t)$.

\subsection{Full-duplex relaying}

For the full-duplex relaying, the received signal at the relay and the destination in time slot $t$ are given by

$$
y_{\mathrm{r}}(t)=h_{\mathrm{sr}} x_{\mathrm{s}}(t)+h_{\mathrm{rr}} x_{\mathrm{r}}(t)+n_{\mathrm{r}}(t),
$$

and

$$
\mathbf{y}_{\mathrm{d}}(t)=\mathbf{h}_{\mathrm{sd}} x_{\mathrm{s}}(t)+\mathbf{h}_{\mathrm{rd}} x_{\mathrm{r}}(t)+\mathbf{n}_{\mathrm{d}}(t),
$$

respectively.

When the AF protocol is used, the processing at the relay is similar to that in the half-duplex mode. As the instantaneous received power at the relay is $E\left[\left|y_{\mathrm{r}}(t)\right|^{2}\right]=$ $P_{\mathrm{s}}\left|h_{\mathrm{sr}}\right|^{2}+P_{\mathrm{r}}\left|h_{\mathrm{rr}}\right|^{2}+\sigma_{\mathrm{r}}^{2}$, the scaling factor is chosen as

$$
\beta=\sqrt{\frac{P_{\mathrm{r}}}{P_{\mathrm{s}}\left|h_{\mathrm{sr}}\right|^{2}+P_{\mathrm{r}}\left|h_{\mathrm{rr}}\right|^{2}+\sigma_{\mathrm{r}}^{2}}} .
$$

Assume the processing delay at the relay is $\tau$ time slot, then the transmit signal at the relay is $x_{\mathrm{r}}(t)=\beta y_{\mathrm{r}}(t-\tau)$.

When the DF protocol is used, the processing at the relay is also similar to that in the half-duplex mode except there is processing delay $\tau$. If the relay can decode the received signal successfully, then the transmit signal at the relay is $x_{\mathrm{r}}(t)=\sqrt{\frac{P_{\mathrm{r}}}{P_{\mathrm{s}}}} x_{\mathrm{S}}(t-\tau)$.

\section{Performance analysis}

In this section, we analyze the performance of the direct transmission, half-duplex relaying, and full-duplex relaying, respectively. We assume matched filter (MF) processing is used at the destination, i.e., the destination processes the signal vector by multiplying the conjugate-transpose of the channel [4]. For large $N$, the channel vectors $\mathbf{h}_{\text {sd }}$ and $\mathbf{h}_{\text {rd }}$ are asymptotically orthogonal, so we have $\mathbf{h}_{\mathrm{sd}}^{H} \mathbf{h}_{\mathrm{sd}} \rightarrow$ $N \eta_{\mathrm{sd}}, \mathbf{h}_{\mathrm{rd}}^{H} \mathbf{h}_{\mathrm{rd}} \rightarrow N \eta_{\mathrm{rd}}, \mathbf{h}_{\mathrm{sd}}^{H} \mathbf{h}_{\mathrm{rd}} \rightarrow 0$ and $\mathbf{h}_{\mathrm{rd}}^{H} \mathbf{h}_{\mathrm{sd}} \rightarrow 0$.

\subsection{Direct transmission}

When MF is used at the destination, we have

$$
\begin{aligned}
\mathbf{h}_{\mathrm{sd}}^{H} \mathbf{y}_{\mathrm{d}}(t) & =\mathbf{h}_{\mathrm{sd}}^{H} \mathbf{h}_{\mathrm{sd}} x_{\mathrm{s}}(t)+\mathbf{h}_{\mathrm{sd}}^{H} \mathbf{n}_{\mathrm{d}}(t) \\
& \approx N \eta_{\mathrm{sd}} x_{\mathrm{s}}(t)+\mathbf{h}_{\mathrm{sd}}^{H} \mathbf{n}_{\mathrm{d}}(t) .
\end{aligned}
$$

Therefore, the achievable rate is given by

$$
R_{\mathrm{DT}}=\log _{2}\left(1+\frac{N P_{\mathrm{s}} \eta_{\mathrm{s}, \mathrm{d}}}{\sigma_{\mathrm{d}}^{2}}\right)=\log _{2}\left(1+N \bar{\gamma}_{\mathrm{s}, \mathrm{d}}\right)
$$

where $\bar{\gamma}_{\mathrm{s}, \mathrm{d}}=P_{\mathrm{s}} \eta_{\mathrm{s}, \mathrm{d}} / \sigma_{\mathrm{d}}^{2}$. 
Outage probability is defined as the probability that the achievable rate falls below a target transmission rate $R_{0}$, so the outage probability can be given by

$$
p_{\mathrm{DT}}^{\text {out }}= \begin{cases}0, & \text { if } R_{D T} \geq R_{0} \\ 1, & \text { if } R_{D T}<R_{0}\end{cases}
$$

\subsection{Half-duplex relaying}

In the odd time slot $t$, the processing at the destination is the same as in (8). In the even time slot $t+1$, by using MF, we have

$$
\begin{aligned}
\mathbf{h}_{\mathrm{rd}}^{H} \mathbf{y}_{\mathrm{d}}(t+1) & =\mathbf{h}_{\mathrm{rd}}^{H} \mathbf{h}_{\mathrm{rd}} x_{\mathrm{r}}(t+1)+\mathbf{h}_{\mathrm{rd}}^{H} \mathbf{n}_{\mathrm{d}}(t+1) \\
& \approx N \eta_{\mathrm{rd}} x_{\mathrm{r}}(t+1)+\mathbf{h}_{\mathrm{rd}}^{H} \mathbf{n}_{\mathrm{d}}(t+1) .
\end{aligned}
$$

We assume the maximum ratio combining (MRC) is applied at the destination, then the end-to-end instantaneous SNR for the AF protocol is given as

$$
\gamma_{\mathrm{HD}, \mathrm{AF}}=N \bar{\gamma}_{\mathrm{sd}}+\frac{N \gamma_{\mathrm{sr}} \bar{\gamma}_{\mathrm{rd}}}{\gamma_{\mathrm{sr}}+N \bar{\gamma}_{\mathrm{rd}}+1},
$$

where $\bar{\gamma}_{\mathrm{rd}}=P_{\mathrm{r}} \eta_{\mathrm{r}, \mathrm{d}} / \sigma_{\mathrm{d}}^{2}$ and $\gamma_{\mathrm{sr}}=P_{\mathrm{s}}\left|h_{\mathrm{sr}}\right|^{2} / \sigma_{\mathrm{r}}^{2}$. The achievable rate for the AF protocol is given as

$$
R_{\mathrm{HD}, \mathrm{AF}}=\frac{1}{2} \log _{2}\left(1+\gamma_{\mathrm{HD}, \mathrm{AF}}\right)
$$

where the factor $1 / 2$ is due to the use of two time slots. The outage probability for the AF protocol is given as

$$
\begin{aligned}
p_{\mathrm{HD}, \mathrm{AF}}^{\mathrm{out}} & =\operatorname{Pr}\left(\frac{1}{2} \log _{2}\left(1+\gamma_{\mathrm{HD}, \mathrm{AF}}\right)<R_{0}\right) \\
& =\operatorname{Pr}\left(\gamma_{\mathrm{HD}, \mathrm{AF}}<\delta_{\mathrm{HD}}\right),
\end{aligned}
$$

where $\delta_{\mathrm{HD}}=2^{2 R_{0}}-1$. If $N \bar{\gamma}_{\mathrm{sd}} \geq \delta_{\mathrm{HD}}$, we have $p_{\mathrm{HD}, \mathrm{AF}}^{\text {out }}=0$; if $N \bar{\gamma}_{\mathrm{sd}}+N \bar{\gamma}_{\mathrm{rd}}<\delta_{\mathrm{HD}}$, we have $p_{\mathrm{HD}, \mathrm{AF}}^{\text {out }}=1$; otherwise, we have

$$
\begin{aligned}
p_{\mathrm{HD}, \mathrm{AF}}^{\text {out }} & =\operatorname{Pr}\left(\gamma_{\mathrm{sr}}<\frac{\left(N \bar{\gamma}_{\mathrm{rd}}+1\right)\left(\delta_{\mathrm{HD}}-N \bar{\gamma}_{\mathrm{sd}}\right)}{N \bar{\gamma}_{\mathrm{rd}}+N \bar{\gamma}_{\mathrm{sd}}-\delta_{\mathrm{HD}}}\right) \\
& =1-\exp \left(-\frac{\left(N \bar{\gamma}_{\mathrm{rd}}+1\right)\left(\delta_{\mathrm{HD}}-N \bar{\gamma}_{\mathrm{sd}}\right)}{\bar{\gamma}_{\mathrm{sr}}\left(N \bar{\gamma}_{\mathrm{sd}}+N \bar{\gamma}_{\mathrm{rd}}-\delta_{\mathrm{HD}}\right)}\right),
\end{aligned}
$$

where $\bar{\gamma}_{\mathrm{sr}}=P_{\mathrm{s}} \eta_{\mathrm{sr}} / \sigma_{\mathrm{r}}^{2}$.

Similarly, the end-to-end instantaneous SNR and achievable rate for the DF protocol are given as

$$
\gamma_{\text {HD,DF }}=\min \left\{\max \left\{\gamma_{\mathrm{sr}}, N \bar{\gamma}_{\mathrm{sd}}\right\}, N \bar{\gamma}_{\mathrm{sd}}+N \bar{\gamma}_{\mathrm{rd}}\right\},
$$
and

$$
R_{\mathrm{HD}, \mathrm{DF}}=\frac{1}{2} \log _{2}\left(1+\gamma_{\mathrm{HD}, \mathrm{DF}}\right)
$$

respectively. The outage probability for the DF protocol is given as

$$
\begin{aligned}
p_{\mathrm{HD}, \mathrm{DF}}^{\text {out }} & =\operatorname{Pr}\left(\frac{1}{2} \log _{2}\left(1+\gamma_{\mathrm{HD}, \mathrm{DF}}\right)<R_{0}\right) \\
& =\operatorname{Pr}\left(\gamma_{\mathrm{HD}, \mathrm{DF}}<\delta_{\mathrm{HD}}\right) .
\end{aligned}
$$

If $N \bar{\gamma}_{\mathrm{sd}} \geq \delta_{\mathrm{HD}}$, we have $p_{\mathrm{HD}, \mathrm{AF}}^{\text {out }}=0$; if $N \bar{\gamma}_{\mathrm{sd}}+N \bar{\gamma}_{\mathrm{rd}}<\delta_{\mathrm{HD}}$, we have $p_{\mathrm{HD}, \mathrm{DF}}^{\text {out }}=1$; otherwise, we have

$$
\begin{aligned}
p_{\mathrm{HD}, \mathrm{DF}}^{\text {out }} & =1-\operatorname{Pr}\left(\gamma_{\mathrm{sr}} \geq \delta_{\mathrm{HD}}\right) \\
& =1-\exp \left(-\frac{\delta_{\mathrm{HD}}}{\bar{\gamma}_{\mathrm{sr}}}\right) .
\end{aligned}
$$

\subsection{Full-duplex relaying}

At the relay, the signal-to-interference-plus-noise ratio (SINR) can be written as

$$
\gamma_{\mathrm{r}}=\frac{P_{\mathrm{s}}\left|h_{\mathrm{sr}}\right|^{2}}{P_{\mathrm{r}}\left|h_{\mathrm{rr}}\right|^{2}+\sigma_{\mathrm{r}}^{2}}
$$

At the destination, by using MF to (6), we can obtain

$$
\begin{aligned}
\mathbf{h}_{\mathrm{sd}}^{H} \mathbf{y}_{\mathrm{d}}(t) & =\mathbf{h}_{\mathrm{sd}}^{H} \mathbf{h}_{\mathrm{sd}} x_{\mathrm{s}}(t)+\mathbf{h}_{\mathrm{sd}}^{H} \mathbf{h}_{\mathrm{rd}} x_{\mathrm{r}}(t)+\mathbf{h}_{\mathrm{sd}}^{H} \mathbf{n}_{\mathrm{d}}(t) \\
& \approx N \eta_{\mathrm{sd}} x_{\mathrm{s}}(t)+\mathbf{h}_{\mathrm{sd}}^{H} \mathbf{n}_{\mathrm{d}}(t), \\
\mathbf{h}_{\mathrm{rd}}^{H} \mathbf{y}_{\mathrm{d}}(t) & =\mathbf{h}_{\mathrm{rd}}^{H} \mathbf{h}_{\mathrm{sd}} x_{\mathrm{s}}(t)+\mathbf{h}_{\mathrm{rd}}^{H} \mathbf{h}_{\mathrm{rd}} x_{\mathrm{r}}(t)+\mathbf{h}_{\mathrm{rd}}^{H} \mathbf{n}_{\mathrm{d}}(t) \\
& \approx N \eta_{\mathrm{rd}} x_{\mathrm{r}}(t)+\mathbf{h}_{\mathrm{rd}}^{H} \mathbf{n}_{\mathrm{d}}(t) .
\end{aligned}
$$

When the AF protocol is used, (22) can be further written as

$$
\begin{aligned}
\mathbf{h}_{\mathrm{rd}}^{H} \mathbf{y}_{\mathrm{d}}(t) \approx & N \eta_{\mathrm{rd}} \beta \mathbf{y}_{\mathrm{r}}(t-\tau)+\mathbf{h}_{\mathrm{rd}}^{H} \mathbf{n}_{\mathrm{d}}(t) \\
= & N \eta_{\mathrm{rd}} \beta\left[h_{\mathrm{s}, \mathrm{r}} x_{\mathrm{s}}(t-\tau)+h_{\mathrm{r}, \mathrm{r}} x_{\mathrm{r}}(t-\tau)\right. \\
& \left.+n_{\mathrm{r}}(t-\tau)\right]+\mathbf{h}_{\mathrm{rd}}^{H} \mathbf{n}_{\mathrm{d}}(t) .
\end{aligned}
$$

The destination can delay (21) by $\tau$ time slots and combine it with (23), then the end-to-end instantaneous equivalent SNR and achievable rate are given as

$$
\gamma_{\mathrm{FD}, \mathrm{AF}}=N \bar{\gamma}_{\mathrm{sd}}+\frac{N \bar{\gamma}_{\mathrm{rd}} \gamma_{\mathrm{r}}}{N \bar{\gamma}_{\mathrm{rd}}+\gamma_{\mathrm{r}}+1},
$$

and

$$
R_{\mathrm{FD}, \mathrm{AF}}=\log _{2}\left(1+\gamma_{\mathrm{FD}, \mathrm{AF}}\right),
$$

respectively. The outage probability is given as

$$
\begin{aligned}
p_{\mathrm{FD}, \mathrm{AF}}^{\text {out }} & =\operatorname{Pr}\left(\log _{2}\left(1+\gamma_{\mathrm{FD}, \mathrm{AF}}\right)<R_{0}\right) \\
& =\operatorname{Pr}\left(\gamma_{\mathrm{FD}, \mathrm{AF}}<\delta_{\mathrm{FD}}\right)
\end{aligned}
$$

where $\delta_{\mathrm{FD}}=2^{R_{0}}-1$. If $N \bar{\gamma}_{\mathrm{sd}} \geq \delta_{\mathrm{FD}}$, we have $p_{\mathrm{FD}, \mathrm{AF}}^{\text {out }}=0$; if $N \bar{\gamma}_{\mathrm{sd}}+N \bar{\gamma}_{\mathrm{rd}}<\delta_{\mathrm{FD}}$, we have $p_{\mathrm{FD}, \mathrm{AF}}^{\text {out }}=1$; otherwise, we have

$$
\begin{aligned}
p_{\mathrm{FD}, \mathrm{AF}}^{\text {out }}= & \operatorname{Pr}\left(\gamma_{\mathrm{r}}<\frac{\left(N \bar{\gamma}_{\mathrm{rd}}+1\right)\left(\delta_{\mathrm{FD}}-N \bar{\gamma}_{\mathrm{sd}}\right)}{N \bar{\gamma}_{\mathrm{sd}}+N \bar{\gamma}_{\mathrm{rd}}-\delta_{\mathrm{FD}}}\right) \\
= & 1-\exp \left(-\frac{\left(N \bar{\gamma}_{\mathrm{rd}}+1\right)\left(\delta_{\mathrm{FD}}-N \bar{\gamma}_{\mathrm{sd}}\right)}{\bar{\gamma}_{\mathrm{sr}}\left(N \bar{\gamma}_{\mathrm{sd}}+N \bar{\gamma}_{\mathrm{rd}}-\delta \mathrm{FD}\right)}\right) \\
& \times \frac{1}{1+\frac{\left(N \bar{\gamma}_{\mathrm{rd}}+1\right)\left(\delta_{\mathrm{FD}}-N \bar{\gamma}_{\mathrm{sd}}\right)}{N \bar{\gamma}_{\mathrm{sd}}+N \bar{\gamma}_{\mathrm{rd}}-\delta_{\mathrm{FD}}}} .
\end{aligned}
$$

where $\bar{\gamma}_{\mathrm{rr}}=P_{\mathrm{r}} \eta_{\mathrm{rr}} / \sigma_{\mathrm{r}}^{2}$. 
Similarly, the end-to-end instantaneous equivalent SNR and achievable rate for the DF protocol are given as

$$
\gamma_{\mathrm{FD}, \mathrm{DF}}=\min \left\{\max \left\{\gamma_{\mathrm{r}}, N \bar{\gamma}_{\mathrm{sd}}\right\}, N \bar{\gamma}_{\mathrm{sd}}+N \bar{\gamma}_{\mathrm{rd}}\right\},
$$

and

$$
R_{\mathrm{FD}, \mathrm{DF}}=\log _{2}\left(1+\gamma_{\mathrm{FD}, \mathrm{DF}}\right),
$$

respectively. The outage probability for the DF protocol is given as

$$
\begin{aligned}
p_{\mathrm{FD}, \mathrm{DF}}^{\text {out }} & =\operatorname{Pr}\left(\log _{2}\left(1+\gamma_{\mathrm{FD}, \mathrm{DF}}\right)<R_{0}\right) \\
& =\operatorname{Pr}\left(\gamma_{\mathrm{FD}, \mathrm{DF}}<\delta_{\mathrm{FD}}\right) .
\end{aligned}
$$

If $N \bar{\gamma}_{\mathrm{sd}} \geq \delta$ FD, we have $p_{\mathrm{FD}, \mathrm{DF}}^{\text {out }}=0$; if $N \bar{\gamma}_{\mathrm{sd}}+N \bar{\gamma}_{\mathrm{rd}}<\delta_{\mathrm{FD}}$, we have $p_{\mathrm{FD}, \mathrm{DF}}^{\text {out }}=1$; otherwise, we have

$$
\begin{aligned}
p_{\mathrm{FD}, \mathrm{DF}}^{\text {out }} & =1-\operatorname{Pr}\left(\gamma_{\mathrm{r}} \geq \delta_{\mathrm{FD}}\right) \\
& =1-\exp \left(-\frac{\delta_{\mathrm{FD}}}{\bar{\gamma}_{\mathrm{sr}}}\right) \frac{\bar{\gamma}_{\mathrm{sr}}}{\bar{\gamma}_{\mathrm{sr}}+\delta_{\mathrm{FD}} \bar{\gamma}_{\mathrm{rr}}} .
\end{aligned}
$$

\subsection{Condition for the superiority of full-duplex relaying over half-duplex relaying}

In this subsection, we derive the condition that full-duplex relaying outperforms half-duplex relaying based on the outage probability. Five cases are considered.

- Case 1: $N \bar{\gamma}_{\mathrm{sd}} \geq \delta_{\mathrm{HD}}$. In this case, we have $p_{\mathrm{HD}, \mathrm{AF}}^{\text {out }}=p_{\mathrm{HD}, \mathrm{DF}}^{\text {out }}=p_{\mathrm{FD}, \mathrm{AF}}^{\text {out }}=p_{\mathrm{FD}, \mathrm{DF}}^{\text {out }}=0$, which means that full-duplex relaying and half-duplex relaying will obtain the same outage performance.

- Case 2: $N \bar{\gamma}_{\mathrm{sd}}+N \bar{\gamma}_{\mathrm{rd}}<\delta_{\mathrm{FD}}$. In this case, we have $p_{\mathrm{HD}, \mathrm{AF}}^{\text {out }}=p_{\mathrm{HD}, \mathrm{DF}}^{\text {out }}=p_{\mathrm{FD}, \mathrm{AF}}^{\text {out }}=p_{\mathrm{FD}, \mathrm{DF}}^{\text {out }}=1$, which means that full-duplex relaying and half-duplex relaying will obtain the same outage performance.

- Case 3: $\delta_{\mathrm{FD}} \leq N \bar{\gamma}_{\mathrm{sd}}<\delta_{\mathrm{HD}}$. In this case, we have $p_{\mathrm{FD}, \mathrm{AF}}^{\text {out }}=p_{\mathrm{FD}, \mathrm{DF}}^{\text {out }}=0$ and $p_{\mathrm{HD}, \mathrm{AF}}^{\text {out }}=p_{\mathrm{HD}, \mathrm{DF}}^{\text {out }}>0$, so full-duplex relaying outperforms half-duplex relaying.

- Case 4: $\delta_{\mathrm{FD}} \leq N \bar{\gamma}_{\mathrm{sd}}+N \bar{\gamma}_{\mathrm{rd}}<\delta_{\mathrm{HD}}$. In this case, we have $p_{\mathrm{HD}, \mathrm{AF}}^{\text {out }}=p_{\mathrm{HD}, \mathrm{DF}}^{\text {out }}=1$ and $p_{\mathrm{FD}, \mathrm{AF}}^{\text {out }}=p_{\mathrm{FD}, \mathrm{DF}}^{\text {out }}<1$, so full-duplex relaying outperforms half-duplex relaying.

- Case 5: $N \bar{\gamma}_{\mathrm{sd}}+N \bar{\gamma}_{\mathrm{rd}} \geq \delta_{\mathrm{HD}}$ and $N \bar{\gamma}_{\mathrm{sd}}<\delta_{\mathrm{FD}}$. In this case, full-duplex relaying outperforms half-duplex relaying when $p_{\mathrm{FD}, \mathrm{AF}}^{\text {out }} \leq p_{\mathrm{HD}, \mathrm{AF}}^{\text {out }}$ for $\mathrm{AF}$ protocol and $p_{\mathrm{FD}, \mathrm{DF}}^{\text {out }} \leq p_{\mathrm{HD}, \mathrm{DF}}^{\text {out }}$ for DF protocol. Using the results (15) and (27), the average residual self-interference for AF protocol should satisfy

$$
\begin{aligned}
\bar{\gamma}_{\mathrm{rr}} \leq & \frac{\bar{\gamma}_{\mathrm{sr}}\left(N \bar{\gamma}_{\mathrm{sd}}+N \bar{\gamma}_{\mathrm{rd}}-\delta_{\mathrm{FD}}\right)}{\left(N \bar{\gamma}_{\mathrm{rd}}+1\right)\left(\delta_{\mathrm{FD}}-N \bar{\gamma}_{\mathrm{sd}}\right)} \\
& \times\left\{\operatorname { e x p } \left[\frac{N \bar{\gamma}_{\mathrm{rd}}\left(N \bar{\gamma}_{\mathrm{rd}}+1\right)}{\bar{\gamma}_{\mathrm{sr}}\left(N \bar{\gamma}_{\mathrm{sd}}+N \bar{\gamma}_{\mathrm{rd}}-\delta_{\mathrm{HD}}\right)}\right.\right. \\
& \left.\left.\times \frac{\left(\delta_{\mathrm{HD}}-\delta_{\mathrm{FD}}\right)}{\left(N \bar{\gamma}_{\mathrm{sd}}+N \bar{\gamma}_{\mathrm{rd}}-\delta_{\mathrm{FD}}\right)}\right]-1\right\} .
\end{aligned}
$$

Using the results (19) and (31), the average residual self-interference for DF protocol should satisfy

$$
\bar{\gamma}_{\mathrm{rr}} \leq \frac{\bar{\gamma}_{\mathrm{sr}}}{\delta_{\mathrm{FD}}}\left[\exp \left(\frac{\delta_{\mathrm{HD}}-\delta_{\mathrm{FD}}}{\bar{\gamma}_{\mathrm{sr}}}\right)-1\right] .
$$

\section{Power control}

In this section, we propose power control to improve the system performance. We assume independent power constraint at both the source and the relay, i.e., $P_{\mathrm{s}} \leq P_{\mathrm{s}}^{\max }$ and $P_{\mathrm{r}} \leq P_{\mathrm{r}}^{\max }$. For half-duplex relaying, it is obvious that the system performance is optimized when the source and the relay use the maximum allowed transmit power, so we focus on power control for full-duplex relaying.

For full-duplex relaying, the source should also use the maximum transmit power because as $P_{\mathrm{s}}$ increases, the achievable rates (25) and (29) increase and the outage probabilities (27) and (31) decrease. As such, we concentrate on power control for the relay. Two scenarios are considered based on the CSI availability.

\subsection{Instantaneous CSI of $\boldsymbol{h}_{\mathrm{sr}}$ and $\boldsymbol{h}_{\mathrm{rr}}$}

In this scenario, we assume the relay has the instantaneous CSI of $h_{\mathrm{sr}}$ and $h_{\mathrm{rr}}$, and the statistical CSI of $\mathbf{h}_{\mathrm{sd}}$ and $\mathbf{h}_{\mathrm{rd}}$, then the power control is to maximize the achievable rates.

For the AF protocol, the power control can be formulated as an optimization problem

$$
\begin{array}{cl}
\underset{P_{\mathrm{r}}}{\operatorname{maximize}} & \frac{N \bar{\gamma}_{\mathrm{rd}} \gamma_{\mathrm{r}}}{N \bar{\gamma}_{\mathrm{rd}}+\gamma_{\mathrm{r}}+1} \\
\text { subject to } & 0<P_{\mathrm{r}} \leq P_{\mathrm{r}}^{\max }
\end{array}
$$

By taking the first derivative of object function with respect to $P_{\mathrm{r}}$ and setting the first derivative equal to zero, we obtain the only one positive root

$$
P_{\mathrm{r}}^{*}=\sqrt{\frac{\sigma_{\mathrm{d}}^{2}}{N \eta_{\mathrm{rd}}}\left(\frac{P_{\mathrm{s}}\left|h_{\mathrm{sr}}\right|^{2}+\sigma_{\mathrm{r}}^{2}}{\left|h_{\mathrm{rr}}\right|^{2}}\right)} .
$$

Therefore, the optimal transmit power of the relay is $P_{\mathrm{r}}^{\mathrm{opt}}=\min \left(P_{\mathrm{r}}^{*}, P_{\mathrm{r}}^{\max }\right)$.

For the DF protocol, the power control can also be formulated as an optimization problem

$$
\begin{array}{ll}
\underset{P_{\mathrm{r}}}{\operatorname{maximize}} & \min \left\{\gamma_{\mathrm{r}}, N \bar{\gamma}_{\mathrm{sd}}+N \bar{\gamma}_{\mathrm{rd}}\right\} \\
\text { subject to } & 0<P_{\mathrm{r}} \leq P_{\mathrm{r}}^{\max }
\end{array}
$$

The optimal solution should satisfy

$$
\frac{P_{\mathrm{s}}\left|h_{\mathrm{sr}}\right|^{2}}{P_{\mathrm{r}}\left|h_{\mathrm{rr}}\right|^{2}+\sigma_{\mathrm{r}}^{2}}=\frac{N P_{\mathrm{s}} \eta_{\mathrm{sd}}}{\sigma_{\mathrm{d}}^{2}}+\frac{N P_{\mathrm{r}} \eta_{\mathrm{rd}}}{\sigma_{\mathrm{d}}^{2}}
$$


By solving this equation, we can obtain the only one positive root

$$
\begin{aligned}
P_{\mathrm{r}}^{*}= & \sqrt{\left(\frac{P_{\mathrm{s}} \eta_{\mathrm{sd}}}{2 \eta_{\mathrm{rd}}}-\frac{\sigma_{\mathrm{r}}^{2}}{2\left|h_{\mathrm{rr}}\right|^{2}}\right)^{2}+\frac{P_{\mathrm{s}}\left|h_{\mathrm{sr}}\right|^{2} \sigma_{\mathrm{d}}^{2}}{N\left|h_{\mathrm{rr}}\right|^{2} \eta_{\mathrm{rd}}}} \\
& -\frac{P_{\mathrm{s}} \eta_{\mathrm{sd}}}{2 \eta_{\mathrm{rd}}}-\frac{\sigma_{\mathrm{r}}^{2}}{2\left|h_{\mathrm{rr}}\right|^{2}} .
\end{aligned}
$$

Similarly, the optimal transmit power of the relay for DF protocol is $P_{\mathrm{r}}^{\mathrm{opt}}=\min \left(P_{\mathrm{r}}^{*}, P_{\mathrm{r}}^{\max }\right)$.

\subsection{Statistical CSI of $\boldsymbol{h}_{\mathrm{sr}}$ and $\boldsymbol{h}_{\mathrm{rr}}$}

In this scenario, we assume the relay only has the statistical CSI of $h_{\mathrm{sr}}, h_{\mathrm{rr}}, \mathbf{h}_{\mathrm{sd}}$, and $\mathbf{h}_{\mathrm{rd}}$, then the power control is to minimize the outage probabilities. Note that power control does not help if $N \bar{\gamma}_{\mathrm{sd}} \geq \delta_{\mathrm{FD}}$ or $N \bar{\gamma}_{\mathrm{sd}}+N \bar{\gamma}_{\mathrm{rd}}<\delta_{\mathrm{FD}}$, so we only need to consider the case that $N \bar{\gamma}_{\mathrm{sd}}<\delta$ FD and $N \bar{\gamma}_{\mathrm{sd}}+N \bar{\gamma}_{\mathrm{rd}} \geq \delta_{\mathrm{FD}}$.

For the AF protocol, minimizing the outage probability (27) is equivalent to minimizing $-\ln \left(1-p_{\mathrm{FD}, \mathrm{AF}}^{\text {out }}\right)$, so the power control can be formulated as an optimization problem

$$
\begin{aligned}
\underset{P_{\mathrm{r}}}{\operatorname{minimize}} & \frac{\left(N \bar{\gamma}_{\mathrm{rd}}+1\right)}{\bar{\gamma}_{\mathrm{sr}}\left(c \bar{\gamma}_{\mathrm{rd}}-1\right)}+\ln \left(1+\frac{\bar{\gamma}_{\mathrm{rr}}\left(N \bar{\gamma}_{\mathrm{rd}}+1\right)}{\bar{\gamma}_{\mathrm{sr}}\left(c \bar{\gamma}_{\mathrm{rd}}-1\right)}\right) \\
\text { subject to } & \frac{\delta_{\mathrm{FD}} \sigma_{\mathrm{d}}^{2}}{\eta_{\mathrm{rd}} N}-\frac{P_{\mathrm{s}} \eta_{\mathrm{sd}}}{\eta_{\mathrm{rd}}}<P_{\mathrm{r}} \leq P_{\mathrm{r}}^{\max }
\end{aligned}
$$

where $c=N /\left(\delta_{\mathrm{FD}}-N \bar{\gamma}_{\mathrm{sd}}\right)$. By taking the first derivative with respect to $P_{\mathrm{r}}$ and setting the first derivative equal to zero, we can obtain $P_{\mathrm{r}}^{*}$. However, it is difficult to find the closed form solution, so we need to use iterative rootfinding algorithms such as the bisection method or the Newton's method to find the numerical solution.

For the DF protocol, to ensure $N \bar{\gamma}_{\mathrm{sd}}+N \bar{\gamma}_{\mathrm{rd}} \geq \delta_{\mathrm{FD}}$, we should set the transmit power at the relay as high as possible. However, after $N \bar{\gamma}_{\mathrm{sd}}+N \bar{\gamma}_{\mathrm{rd}} \geq \delta_{\mathrm{FD}}$ is satisfied, the outage probability increases as $P_{\mathrm{r}}$ increases. Therefore, the optimal transmit power of the relay is

$$
P_{\mathrm{r}}^{*}=\frac{\delta_{\mathrm{FD}} \sigma_{\mathrm{d}}^{2}}{\eta_{\mathrm{rd}} N}-\frac{P_{\mathrm{s}} \eta_{\mathrm{sd}}}{\eta_{\mathrm{rd}}} .
$$

\section{Numerical results}

In this section, we present some numerical results to validate our analysis. For simplicity, we assume $N=100$, $P_{\mathrm{s}}^{\max }=1, P_{\mathrm{r}}^{\max }=1, \eta_{\mathrm{sd}}=10^{-7}, \eta_{\mathrm{rd}}=2 \times 10^{-6}$, $\sigma_{\mathrm{d}}^{2}=\sigma_{\mathrm{r}}^{2}=10^{-6}$, and $R_{0}=5 \mathrm{bps} / \mathrm{Hz}$.

In Fig. 2, we plot the achievable rates as a function of $\gamma_{\mathrm{sr}}$ for direct transmission, half-duplex relaying, and fullduplex relaying, where $\left|h_{\mathrm{rr}}\right|^{2}=4 \times 10^{-6}$. From Fig. 2, we can see that (1) the achievable rates of both half-duplex relaying and full-duplex relaying increase as $\gamma_{\mathrm{sr}}$ increases; (2) the full-duplex AF relaying perform better than fullduplex DF in the low SNR regime, but perform worse than full-duplex DF in the high SNR regime; and (3) the achievable rates of full-duplex relaying can be improved by using power control.

In Fig. 3, we plot the achievable rates as a function of $\left|h_{\mathrm{rr}}\right|^{2}$ for direct transmission, half-duplex relaying, and full-duplex relaying, where $\gamma_{\mathrm{sr}}=30 \mathrm{~dB}$. It is observed from Fig. 3 that as $\left|h_{\mathrm{rr}}\right|^{2}$ increases, the achievable rates of

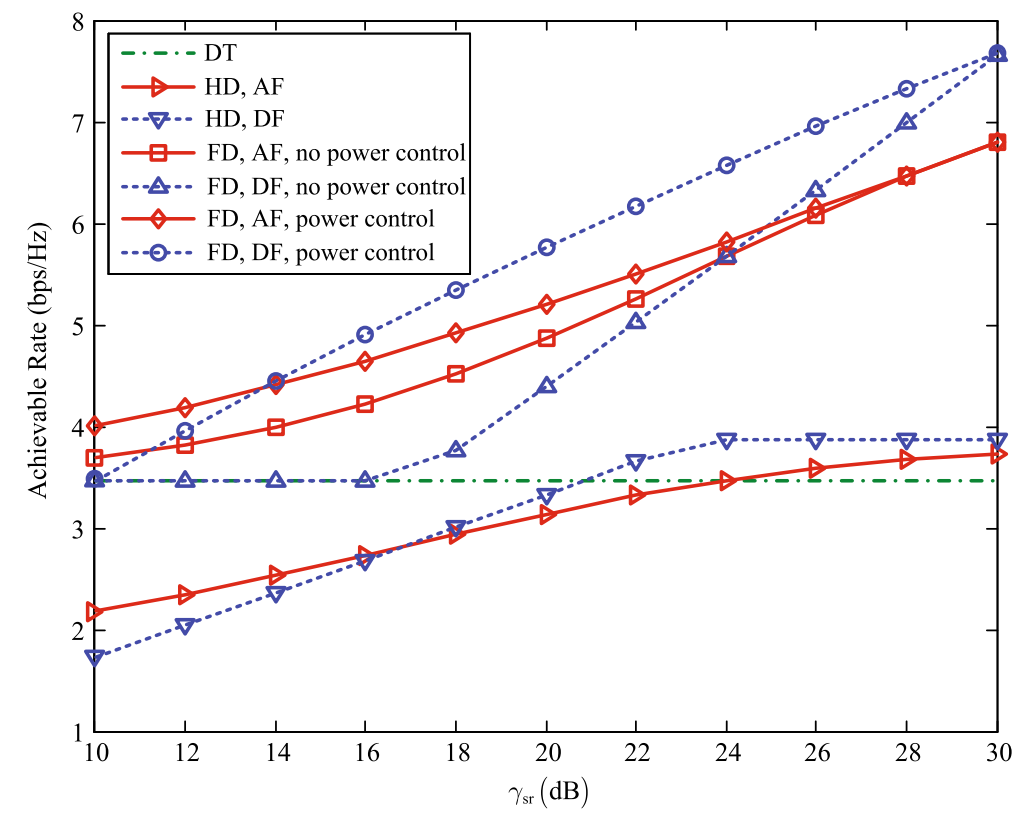

Fig. 2 Achievable rates versus $\gamma_{\mathrm{sr}}$ for direct transmission, half-duplex relaying, and full-duplex relaying, with $\left|h_{\mathrm{rr}}\right|^{2}=4 \times 10^{-6}$ 


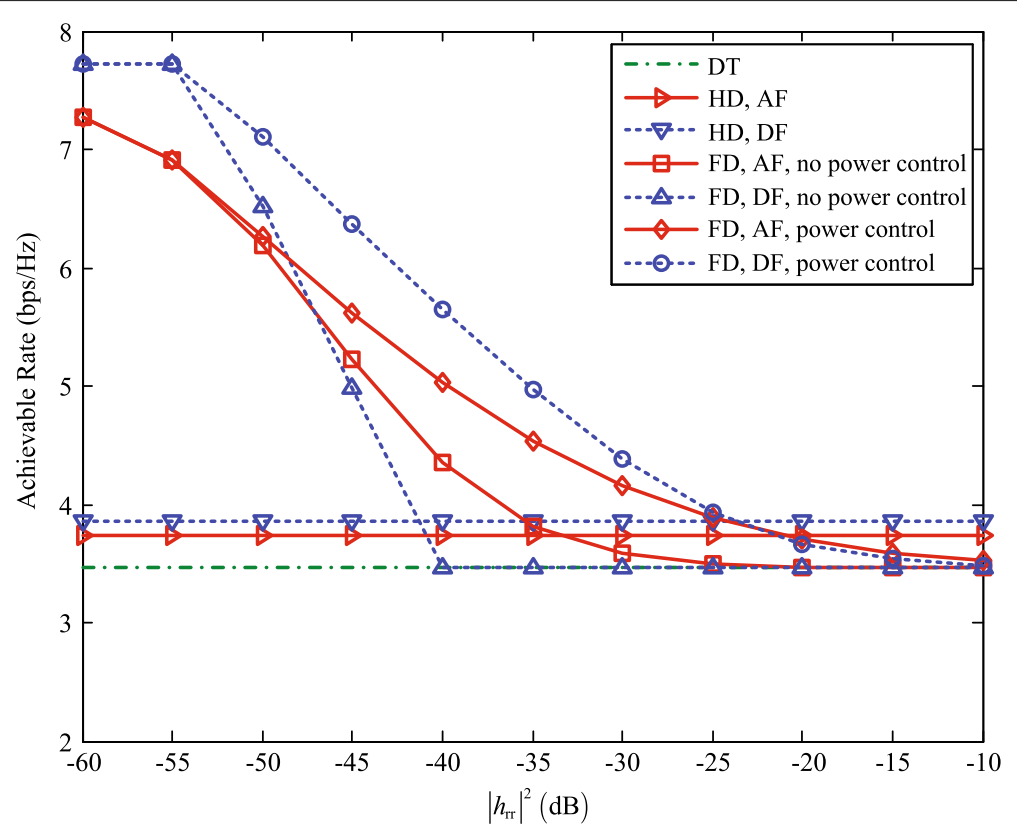

Fig. 3 Achievable rates versus $\left|h_{\mathrm{rr}}\right|^{2}$ for direct transmission, half-duplex relaying, and full-duplex relaying, with $\gamma_{\mathrm{sr}}=30 \mathrm{~dB}$

full-duplex relaying decrease significantly. When $\left|h_{\mathrm{rr}}\right|^{2}$ is high enough, the achievable rates of half-duplex relaying will outperform that of full-duplex relaying. Moreover, we can also see that power control can improve the achievable rates of full-duplex relaying.

Figure 4 shows the outage probabilities versus $\bar{\gamma}_{\mathrm{sr}}$ for full-duplex relaying with $E\left(\left|h_{\mathrm{rr}}\right|^{2}\right)=4 \times 10^{-6}$, while
Fig. 5 shows the outage probabilities versus $E\left(\left|h_{\mathrm{rr}}\right|^{2}\right)$ for full-duplex relaying with $\bar{\gamma}_{\mathrm{sr}}=40 \mathrm{~dB}$. Note that $N P_{\mathrm{s}}^{\max } \eta_{\mathrm{sd}} / \sigma_{\mathrm{d}}^{2}+N P_{\mathrm{r}}^{\max } \eta_{\mathrm{rd}} / \sigma_{\mathrm{d}}^{2}<\delta_{\mathrm{HD}}$, so the outage probabilities for half-duplex relaying equal to 1 . From Figs. 4 and 5, we can see that (1) the outage probabilities of full-duplex relaying decrease as $\bar{\gamma}_{\mathrm{sr}}$ increases and increase as $E\left(\left|h_{\mathrm{rr}}\right|^{2}\right)$ increases; (2) the full-duplex DF relaying with

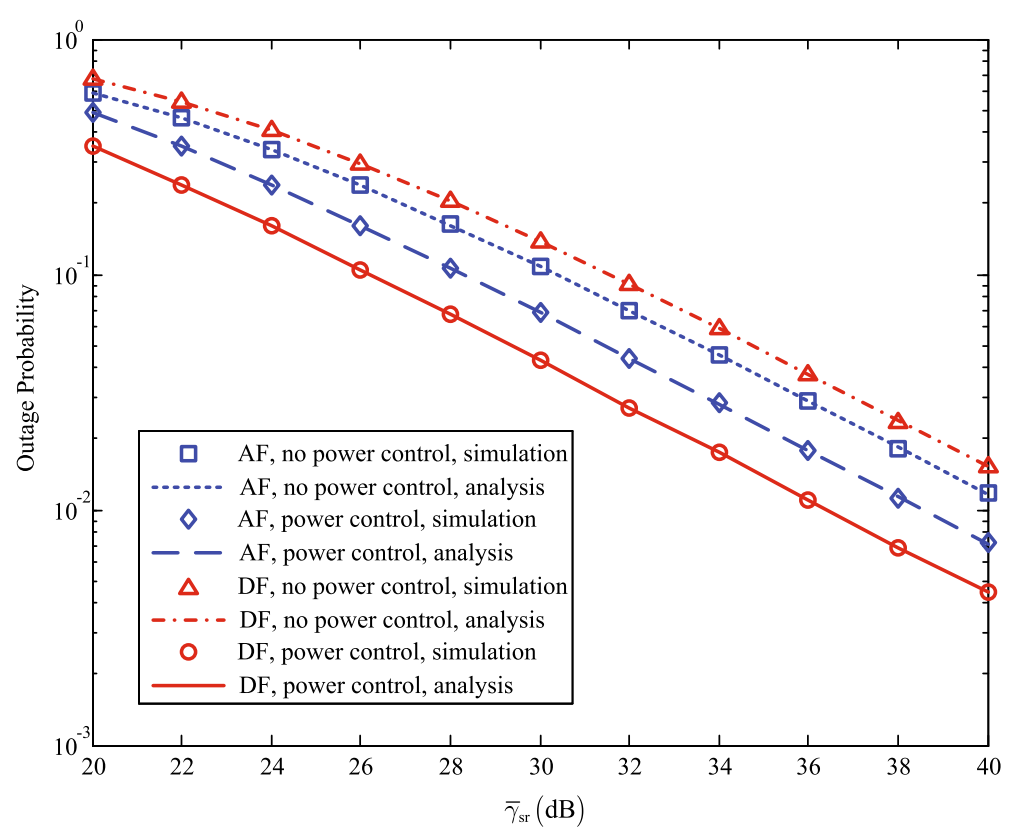

Fig. 4 Outage probability versus $\bar{\gamma}_{\mathrm{sr}}$ for full-duplex relaying, with $E\left(\left|h_{\mathrm{rr}}\right|^{2}\right)=4 \times 10^{-6}$ 


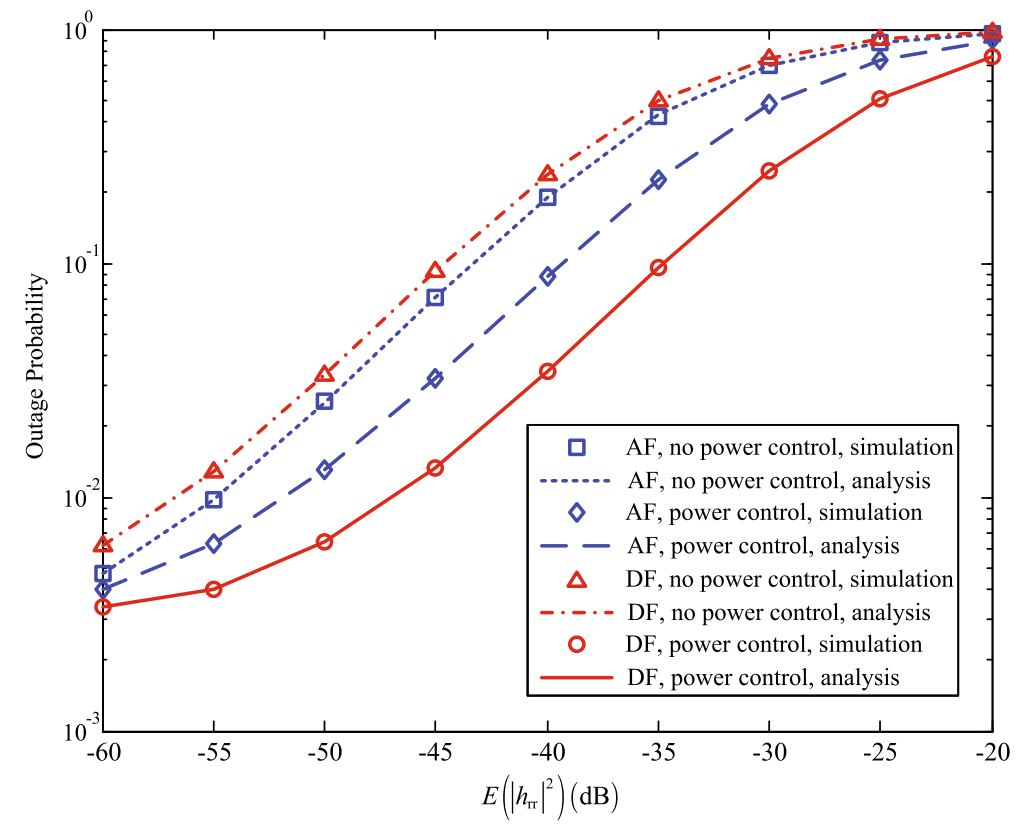

Fig. 5 Outage probability versus $E\left(\left|h_{\mathrm{rr}}\right|^{2}\right)$ for full-duplex relaying, with $\bar{\gamma}_{\mathrm{sr}}=40 \mathrm{~dB}$

power control will obtain the best performance and the full-duplex DF relaying without power control will obtain the worst performance; and (3) the outage probabilities of full-duplex relaying can be improved by using power control.

\section{Conclusions}

In traditional full-duplex relaying, the destination always treats the source-destination link as interference, which degrades the system performance. In this paper, we investigated the system performance of full-duplex relaying by considering large-scale antenna array at the destination. Using the property that the source-destination channel vector and relay-destination channel vector become pairwise orthogonal, we converted the source-destination link to useful signal. We derived the achievable rates and outage probabilities for direct transmission, halfduplex relaying, and full-duplex relaying, respectively. By maximizing the achievable rates or minimizing the outage probabilities based on the CSI availability, we obtained the optimal transmit power of the full-duplex relay node. Numerical results showed that the performance of full-duplex relaying is determined by the residual self-interference, and power control can improve the performance of full-duplex relaying.

\section{Acknowledgements}

This work was supported by the 863 Program of China (2015AA01A703), the Fundamental Research Funds for the Central Universities (2014ZD03-02), the National Natural Science Foundation of China (61571055), the China Scholarship Council (201508120012), and the Doctoral Foundation of Tianjin Normal University (5RL135).

\section{Competing interests}

The authors declare that they have no competing interests.

\section{Author details}

${ }^{1}$ Tianjin Key Laboratory of Wireless Mobile Communications and Power Transmission, Tianjin Normal University, Tianjin 300387, China. ${ }^{2}$ College of Electronic and Communication Engineering, Tianjin Normal University, Tianjin 300387, China. ${ }^{3}$ Key Lab of Universal Wireless Communications, MOE, Beijing University of Posts and Telecommunications, Beijing 100876, China.

Received: 31 December 2016 Accepted: 21 February 2017

Published online: 28 February 2017

\section{References}

1. JG Andrews, S Buzzi, W Choi, SV Hanly, et al., What will 5 G be?. IEEE J. Sel. Areas Commun. 32(6), 1065-1082 (2014)

2. M Agiwal, A Roy, N Saxena, Next generation $5 \mathrm{G}$ wireless networks: a comprehensive survey. IEEE Commun. Surveys Tuts. 18(3), 1617-1655 (2016)

3. F Boccardi, RW Heath, A Lozano, TL Marzetta, P Popovski, Five disruptive technology directions for 5G. IEEE Commun. Mag. 52(2), 74-80 (2014)

4. F Rusek, D Persson, BK Lau, EG Larsson, TL Marzetta, et al., Scaling up MIMO: opportunities and challenges with very large arrays. IEEE Signal Process. Mag. 30(1), 40-60 (2013)

5. E Larsson, O Edfors, F Tufvesson, TL Marzetta, Massive MIMO for next generation wireless systems. IEEE Commun. Mag. 52(2), 186-195 (2014)

6. L Lu, GY Li, AL Swindlehurst, A Ashikhmin, Z Rui, An overview of massive MIMO: benefits and challenges. IEEE J. Sel. Topics Signal Process. 8(5), 742-758 (2014)

7. TL Marzetta, Noncooperative cellular wireless with unlimited numbers of base station antennas. IEEE Trans. Wireless Commun. 9(11), 3590-3600 (2010)

8. H Yang, TL Marzetta, Performance of conjugate and zero-forcing beamforming in large-scale antenna systems. IEEE J. Sel. Areas Commun. 31(2), 172-179 (2013)

9. HQ Ngo, EG Larsson, TL Marzetta, Energy and spectral efficiency of very large multiuser MIMO systems. IEEE Trans. Commun. 61(4), 1436-1449 (2013)

10. X Gao, O Edfors, F Rusek, F Tufvesson, Massive MIMO performance evaluation based on measured propagation data. IEEE Trans. Wireless Commun. 14(7), 3899-3911 (2015) 
11. S Hong, J Brand, C Jung, et al., Applications of self-interference cancellation in $5 \mathrm{G}$ and beyond. IEEE Commun. Mag. 52(2), 114-121 (2014)

12. M Heino, D Korpi, T Huusari, et al., Recent advances in antenna design and interference cancellation algorithms for in-band full duplex relays. IEEE Commun. Mag. 53(5), 91-101 (2015)

13. H Ju, E Oh, D Hong, Improving efficiency of resource usage in two-hop full duplex relay systems based on resource sharing and interference cancellation. IEEE Trans. Wireless Commun. 8(8), 3933-3938 (2009)

14. T Kwon, S Lim, S Choi, D Hong, Optimal duplex mode for DF relay in terms of the outage probability. IEEE Trans. Veh. Techol. 59(7), 3628-3634 (2010)

15. TRiihonen, S Werner, R Wichman, Optimized gain control for single-frequency relaying with loop interference. IEEE Trans. Wireless Commun. 8(6), 2801-2806 (2009)

16. TRiihonen, S Werner, R Wichman, Mitigation of loopback self-interference in full-duplex MIMO relays. IEEE Trans. Signal Process. 59(12), 5983-5993 (2011)

17. T Riihonen, S Werner, R Wichman, Hybrid full-duplex/half-duplex relaying with transmit power adaptation. IEEE Trans. Wireless Commun. 10(9), 3074-3085 (2011)

18. TK Baranwal, DS Michalopoulos, R Schober, Outage analysis of multihop full duplex relaying. IEEE Commun. Lett. 17(1), 63-66 (2013)

19. $B$ Yu, L Yang, X Cheng, R Cao, Power and location optimization for full-duplex decode-and-forward relaying. IEEE Trans. Commun. 63(12), 4743-4753 (2015)

20. HQ Ngo, HA Suraweera, M Matthaiou, EG Larsson, Multipair full-duplex relaying with massive arrays and linear processing. IEEE J. Sel. Areas Commun. 32(9), 1721-1737 (2014)

21. XXia, D Zhang, KXu, et al., Hardware impairments aware transceiver for full-duplex massive MIMO relaying. IEEE Trans. Signal Process. 63(24), 6565-6580 (2015)

\section{Submit your manuscript to a SpringerOpen ${ }^{\circ}$ journal and benefit from:}

- Convenient online submission

- Rigorous peer review

- Immediate publication on acceptance

- Open access: articles freely available online

- High visibility within the field

Retaining the copyright to your article

Submit your next manuscript at $\gg$ springeropen.com 